\title{
Calculating the proportion of avoidable attendances at UK emergency departments: analysis of the Royal College of Emergency Medicine's Sentinel Site Survey data
}

\author{
Toby Morris, ${ }^{1}$ Suzanne M Mason, ${ }^{2}$ Chris Moulton, ${ }^{3}$ Colin O'Keeffe $^{2}$
}

\begin{abstract}
- Additional material is published online only. To view please visit the journal online (http://dx.doi.org/10.1136/ emermed-2017-206846).

${ }^{1}$ Medical School, University of Sheffield, Sheffield, UK

${ }^{2} S c h o o l$ of Health and Related Research, University of Sheffield, Sheffield, UK

${ }^{3}$ Department of Emergency, Royal Bolton Hospital, Bolton, UK
\end{abstract}

Correspondence to Dr Toby Morris, Medical School, University of Sheffield, Sheffield S10 2RX, UK

tobymorris@doctors.net.uk

Received 21 April 2017

Revised 6 July 2017

Accepted 28 August 2017

Published Online First

30 October 2017

\section{Linked}

- http://dx.doi.org/10.1136/ emermed-2017-206953

Check for updates

To cite: Morris T

Mason SM, Moulton C,

et al. Emerg Med J

2018:35:114-119.

\begin{abstract}
Introduction Avoidable attendances (AAs; defined as non-urgent, self-referred patients who could be managed more effectively and efficiently by other services) have been identified as a contributor to ED crowding. Internationally, AAs have been estimated to constitute $10 \%-90 \%$ of ED attendances, with the UK 2013 Urgent and Emergency Care Review suggesting a figure of $40 \%$. Methods This pilot study used data from the Royal College of Emergency Medicine's Sentinel Site Survey to estimate the proportion of AAs in 12 EDs across England on a standard day (20 March 2014). AAs were defined by an expert panel using questions from the survey. All patients attending the EDs were recorded with details of investigations and treatments received, and the proportion of patients meeting criteria for AA was calculated.
\end{abstract}

Results Visits for 3044 patients were included. Based on these criteria, a mean of $19.4 \%(95 \% \mathrm{Cl} 18.0 \%$ to $20.8 \%$ ) of attendances could be deemed avoidable. The lowest proportion of AAs reported was $10.7 \%$, while the highest was $44.3 \%$. Younger age was a significant predictor of AA with mean age of 38.6 years for all patients attending compared with 24.6 years for patients attending avoidably ( $\mathrm{p} \leq 0.001)$.

Discussion The proportion of AAs in this study was lower than many estimates in the literature including that reported by the 2013 Urgent and Emergency Care Review. This suggests the ED is the most appropriate healthcare setting for many patients due to comprehensive investigations, treatments and capability for urgent referrals. The proportion of AAs is dependent on the defining criteria used, highlighting the need for a standardised, universal definition of an appropriate/avoidable ED attendance. This is essential to understanding how AAs contribute to the overall issue of crowding.

\section{INTRODUCTION}

The challenges faced by EDs around the world are constantly debated in the political and medical environments. One such challenge is that the number of patients presenting to the ED has the potential to outweigh the limited resources, staff hours and space within the department, leading to crowding, exit block and decreased performance against targets such as the UK 4-hour standard for time spent in the ED. A growing international body of literature documents the adverse effects

\section{Key messages}

What is already known on this subject

- Avoidable ED attendances are thought to contribute to crowding, which has welldocumented negative effects on patient care. The cost-effectiveness of programmes to divert avoidable visits, however, depends of how often these visits occur. There is a wide range in estimations of avoidable attendances with no standardised definition; international systematic reviews have reported avoidable attendances vary from $10 \%$ to $90 \%$ of all ED visits. The NHS England Urgent and Emergency Care Review (2013) suggests around 40\% of patients do not need treating in the ED and could be more effectively looked after in the community.

\section{What this paper adds}

- In this prospective, multicentre pilot study, we determined that $19.4 \%$ of attendances could be deemed avoidable, using criteria developed by a panel of experts and clinicians from the Royal College of Emergency Medicine. This figure, lower than the estimate of the Urgent and Emergency Care Review 2013, suggests that the ED remains the most appropriate healthcare setting for most patients and that attempting to redirect patients from the ED to the community may often not a suitable solution. A standardised definition of an avoidable/ appropriate attendance is needed in order to conduct further research in this field.

of crowding in the ED, including, but not limited to: increased patient mortality and adverse health outcomes, increased numbers of patients leaving without being seen and increased patient perceptions of compromised care. ${ }^{1-4}$ Data published in 2016 by the Nuffield Trust and The Health Foundation show the lowest performance on record against the UK 4-hour operational standard in the fourth quarter of 2015/2016 (81.81\%), indicating EDs are struggling more than ever under the weight of increased demand and finite resources. ${ }^{5}$

One factor that may be increasing pressure on the ED is patients using its services unnecessarily or the so-called avoidable attendances (AAs). These 
attendances, also referred to as 'preventable', 'unnecessary' or 'inappropriate', are said to have a number of undesirable effects on urgent and emergency care in EDs, including increased waiting times, delays in treatment for other patients, impaired access and significant financial implications for commissioners, service providers and patients themselves. ${ }^{6}{ }^{7}$ However, the overall significance of AAs is disputed, with studies by other authors stating that AAs contribute minimally to crowding and the associated negative effects. ${ }^{8}$

There is no standardised definition of an AA; however, an avoidable attendee is typically described as a self-referred, low urgency patient who could be managed more effectively and efficiently by other healthcare service providers. ${ }^{6}$ Systematic reviews on the subject of AAs have reported prevalence ranging from $10 \%$ to $90 \%$, the large variation possibly due to the lack of a single, standardised definition of 'avoidability'. ${ }^{9}$ Documented methods of determining AAs include subjective clinical opinion, ${ }^{9}{ }^{10}$ pre-existing triage scores ${ }^{9}{ }^{11-13}$ and independently developed clinical criteria. ${ }^{69}$ Results vary greatly, even when similar methods are applied. Dinh et al report a difference of $14 \%$ in the number 'GP patients in the ED' when applying the Australian Institute of Health and Welfare definition versus the Australian College of Emergency Medicine definition, despite both being based on predetermined triage scores. ${ }^{11} 13$

Understanding what proportion of patients are attending the ED avoidably and which patients are likely to do so, is essential to further understanding whether AAs significantly contribute to the overall issue of crowding. In the UK, reports such as NHS England's 2013 Urgent and Emergency Care Review have strongly implied that $40 \%$ of attendances at EDs are avoidable (though exact methodology is not explained) and that redirecting these patients to other services, such as primary care, walk-in centres and minor injuries units, would help to alleviate the problem of crowding. ${ }^{1415}$

The primary aim of the Sentinel Site Survey (SSS), conducted by the UK Royal College of Emergency Medicine (RCEM), was to determine the proportion of patients attending a sample of EDs across England that could be deemed avoidable, that is, patients who could be treated adequately in a less urgent setting such as tertiary acute care services or primary care. This study was designed as a small-scale pilot to test methodology of data collection and analysis, prior to conducting similar larger scale studies in the future.

\section{METHODS}

The SSS was undertaken by the RCEM on Thursday, 20 March 2014 with the aim of capturing a standard day in the participating EDs. Information on method of arrival, treatments/ investigations received, age of patients and suitability to be seen by a GP was collected, as well as information on staffing levels in departments, which was not included in this study. The SSS provided a source of data, independent to NHS England, that when analysed could help to determine the proportion of patients attending the ED who could be treated in a less urgent setting and thus the overall significance of AAs in EDs.

A convenience sample of 12 EDs from across England took part in the survey on a voluntary basis, varying in size, location and case mix. A selection of type 1 departments in England, defined as major departments providing consultant-led 24-hour services with full resuscitation facilities, ${ }^{16}$ were invited to participate by a panel of expert clinicians from the RCEM. Departments who responded within a designated time frame were included. All departments who responded were included in the
Box 1 Questions included in the Sentinel Site Survey

1. Age of patient

2. Did the patient arrive by ambulance?*

3. Was an ECG done?*

4. Were any blood tests taken? (not stick for glucose)*

5. Were any X-rays or other diagnostic imaging done?*

6. Were any parenteral drugs/fluids or immunisations administered?*

7. Was any physical treatment administered?*

8. Were any prescriptions issued?

9. Was patient admitted to hospital or other facility for an overnight stay?*

10. Was the patient referred urgently to a clinic or other outpatient facility?*

11. In your opinion, could this patient have been dealt with by a general practitioner (GP) working in your ED?

12. If answer to question $11=y e s$, please answer question 12

13. If you had the ability at triage to book this patient an appointment with a GP within the next 24 hours, using only the information available at triage would this have been both safe and clinically appropriate?

*Questions highlighted with an asterisk were used to determine appropriate/avoidable attendance.

study, and instructions on how data were to be collected were sent to a lead clinician in each department who acted as a point of contact. The final sample included major trauma centres, teaching hospitals and district general hospitals. Further details on departments involved is included in online supplementary appendix 1 .

Senior consultants within the EDs were instructed to complete the questions included in the survey for all patients attending the ED between 00.00 and 23.59 hours on the date in question, with the exception of patients who left before being seen (recorded in a separate part of the survey). In order to complete the data collection, consultants were required to analyse individual patient records and to enter data retrospectively into the survey. Any patient-identifying details were omitted in the interests of confidentiality. Selection bias was minimised in this study by the wide variation in the size and location of EDs participating.

The SSS was developed with the aim of collecting a minimum dataset of information on each patient attending and consisted of 12 questions for each patient. Questions were developed by an expert group of emergency medicine consultants convened through the RCEM defining avoidability based on processes of care, a method previously shown to be more accurate than triage or diagnostic codes by a previous study on AAs. ${ }^{17}$ The questions are shown in box 1 .

Answering 'yes' to one or more of the highlighted questions meant the attendance was deemed appropriate. The criteria were selected as it was felt that they could not be administered or carried out rapidly in most primary care settings. In the case of 'arrival by ambulance', it was considered that although patients may be using the ambulance service unnecessarily, once in an ambulance, a patient will almost invariably be transported to an ED. Receiving a prescription, in the absence of other investigations or treatments, was felt not to be an adequate reason to be seen in the ED. Questions 11 and 12 were not included in the analysis; this is discussed further in limitations. 


\section{Outcome}

The primary outcome was the proportion of visits that met criteria for an avoidable visit according to one or more of the eight criteria. The secondary outcome measured was the possible association of age with AAs, in order to determine whether potential interventions to reduce AAs would be best aimed at specific age groups.

\section{Analysis}

Data were initially entered by each participating ED into a Microsoft Excel spreadsheet. The spreadsheets from each department were then collated into one document and imported into IBM SPSS V.22 9.5.0.0 for analysis. Data cleaning was performed to identify and remove patient records with missing data. If enough data were present in an entry to determine whether the attendance was avoidable or appropriate, the entry was kept within the dataset, and if not, it was removed.

Patient records were then computed into binary data with each entry receiving a score of 1 if an 'appropriate' variable was present, and conversely a score of 0 if no 'appropriate' variables were present. This allowed the calculation of the proportion of patients felt to be attending appropriately. Sample size calculations (using a predicted value of 40\% from the 2013 Urgent and Emergency Care Review ${ }^{14}$ ) showed that a sample size of greater than 369 patient episodes were need to ensure confidence intervals of \pm 0.05 . $^{18}$

\section{RESULTS}

Three thousand and fifty-eight (3058) patients were included in the survey. Patients with incomplete data to the extent where the attendance could not be identified as appropriate or avoidable were excluded from the dataset (14 cases). This left complete data for 3044 patients, surpassing the sample size required to ensure adequate power by a factor of more than eight.

A cross-tabulation of departments and the avoidable/appropriate variable was undertaken to show the proportion of AAs at each department using the criteria in box 1 . The results are presented in table 1 and figure 1 below:

Table 1 and figure 1 demonstrate that using these defining criteria, the data in the SSS estimated the mean proportion of AAs to be $19.4 \%$ (95\% CI $18.0 \%$ to $20.8 \%$ ) of total ED attendances. Sheffield Northern General Hospital reported the lowest proportion of AAs at $10.7 \%$, while Birmingham Children's Hospital reported the highest at $44.3 \%$, considerably greater than other departments. The former result is from an adult only
$\mathrm{ED}$, while the latter is from a paediatric ED, strongly suggesting that these values are likely to be case mix related.

\section{Analysis of results by age}

The ages of patients were recorded in the SSS, allowing attendances to be analysed using this variable. Ages were divided into: aged $\leq 15$ years, aged $16-64$ years and aged $\geq 65$ years to broadly represent children, adults and older patients. Figure 2 shows the proportion of total attendances from each age group.

Figure 2 shows that adults aged 16-64 years constituted the majority of attendances $(57.1 \%, 1738$ patients on the day of the survey) as expected, due to this being the widest age range. The mean age of all patients attending the ED on this day was 38.6 years (95\% CI 37.6 to 39.5 ), while the mean age of patients attending avoidably was 24.6 (95\% CI 22.9 to 26.2), a difference shown to be statistically significant ( $p \leq 0.001$ on MannWhitney U test).

Patients under 16 years and over 65 years constitute similar proportions of attendances: 22.6\% (690 patients) and 20.3\% (616 patients), respectively. Although adults aged 16-64 years form the majority of ED attendances in this study, a clear inverse association can be seen between age and proportion of AAs. A percentage of 34.9 (95\% CI $31.3 \%$ to $38.5 \%$ ) of patients under 16 years were considered to be attending avoidably, compared with $18.4 \%$ (95\% CI $16.6 \%$ to $20.2 \%$ ) of adults aged $16-65 \%$ and $5.0 \%$ (95\% CI $3.3 \%$ to $6.8 \%)$ of patients over 65 years.

\section{DISCUSSION}

Our study, and method of defining avoidability, has shown that the mean proportion of AAs on the day of the survey was $19.4 \%$ (95\% CI $18.0 \%$ to $20.8 \%$ ) of total attendances across the 12 EDs included in the SSS. In their 2009 systematic review, Carret et al report the prevalence of AAs to range from 10\% to $90 \%$ (possibly due to a wide range of methodologies and definitions of 'avoidability'), ${ }^{9}$ therefore our figure can be viewed as being relatively low (though it is worth noting nearly half the studies included in the systematic review reported more conservative prevalences ranging from $24 \%$ to $40 \%$ ). Overall proportions of AAs reported by EDs ranged from $10.7 \%$ to $44.3 \%$ (as shown in table 1).

The initial (2013) report of the Urgent and Emergency Care Review stated that $40 \%$ of patients are discharged from the ED requiring no treatment and concluded that patient care should be 'shifted' away from the hospital and into the community. ${ }^{14}$ However, it is very important to recognise that receiving no

Table 1 Total number of attendances and AAs at participating EDs

\begin{tabular}{lllll}
\hline & Case mix & Total number of attendances & N (\%) of avoidable attendances (AAs) \\
\hline Department & Taunton, Musgrove Park & Adults and children & 125 & $20(16.0)$ \\
& Leeds, St. James & Adults & 239 & $28(11.7)$ \\
& Bath, Royal United & Adults and children & 189 & $32(16.9)$ \\
& Portsmouth, Queen Alexandra & Adults and children & 246 & $44(17.9)$ \\
& Sheffield Northern General Hospital & Adults & 289 & $31(10.7)$ \\
& Norfolk and Norwich & Adults and children & 274 & $47(17.2)$ \\
& Leeds General Infirmary & Adults and children & 304 & $65(21.4)$ \\
Middlesbrough, James Cook & Adults and children & 279 & $49(17.6)$ \\
& London, St. Thomas & Adults and children & 365 & $81(22.2)$ \\
& Dudley, Russell's Hall & Adults and children & 266 & $55(20.7)$ \\
Bolton, Royal Bolton & Adults and children & 328 & $77(23.4)$ \\
& Birmingham Children's Hospital & Children & 140 & $62(44.3)$ \\
& Total & 3044 & $591(19.4)$ \\
\hline
\end{tabular}




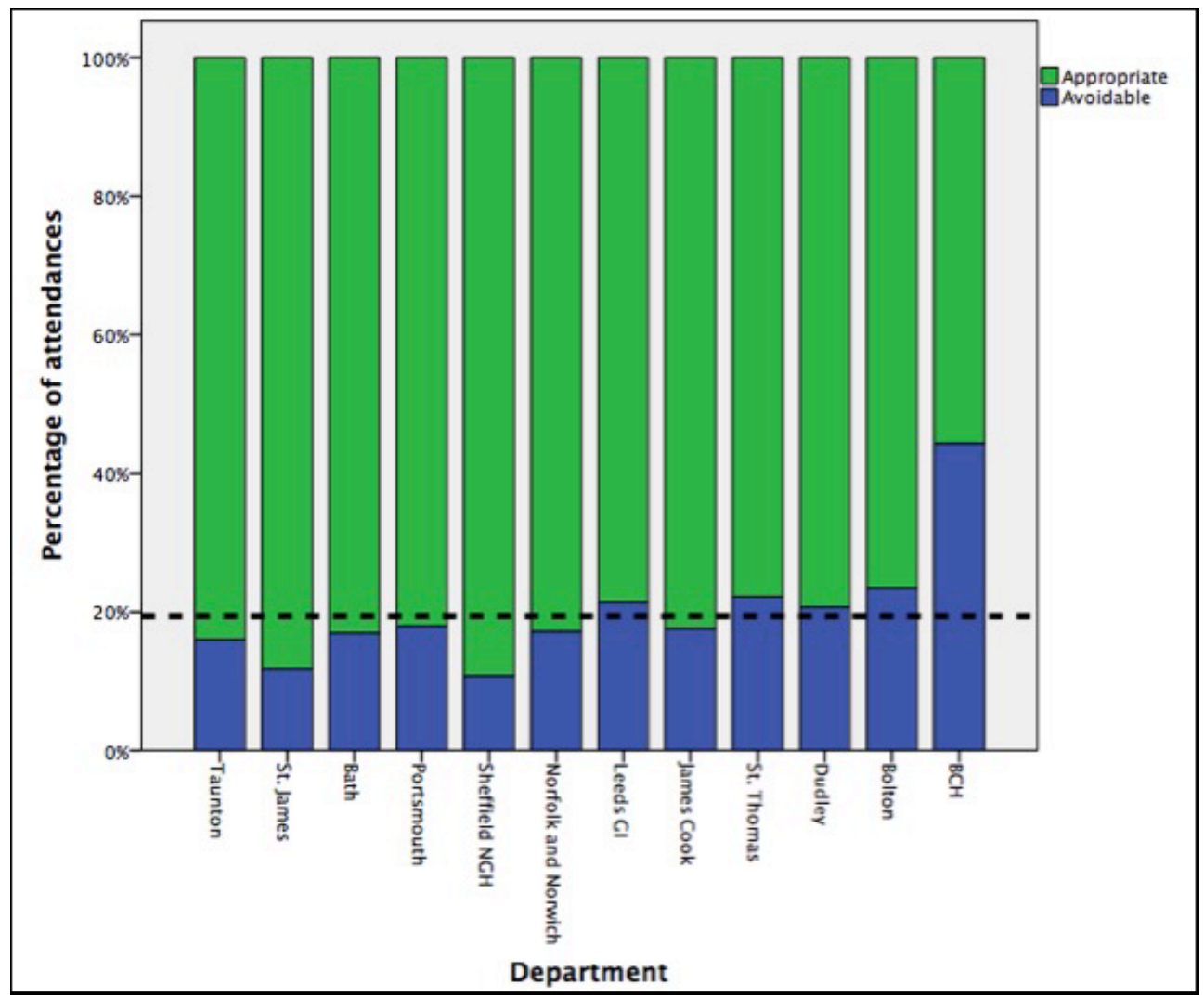

Figure 1 Stacked bar chart showing the proportion of avoidable/appropriate attendances by department (black line representing the mean proportion of AAs across all department). AAs, avoidable attendances; GI, General Infirmary; NGH, Northern General Hospital.

treatment is not the same as not requiring the services of an ED. Whereas the Review claimed that 'untreated' patients could be seen closer to home, this current study took other factors into account, such as the need for investigations and onward urgent referral, as valid reasons for a patient to attend the ED. Using the SSS data, this method produced the much lower figure of $19.4 \%$ as the average proportion of AAs. The statistic of $40 \%$ of patients not requiring treatment in the ED is not present in the final, revised version of the Urgent and Emergency Care Review published in 2015 . $^{19}$

The apparent inverse association between age and AAs (highlighted by figure 2) is replicated in other literature ${ }^{91320-23}$ and is consistent with McHale et al's ${ }^{6}$ conclusion that interventions to reduce AAs would be best targeted at parents of young children

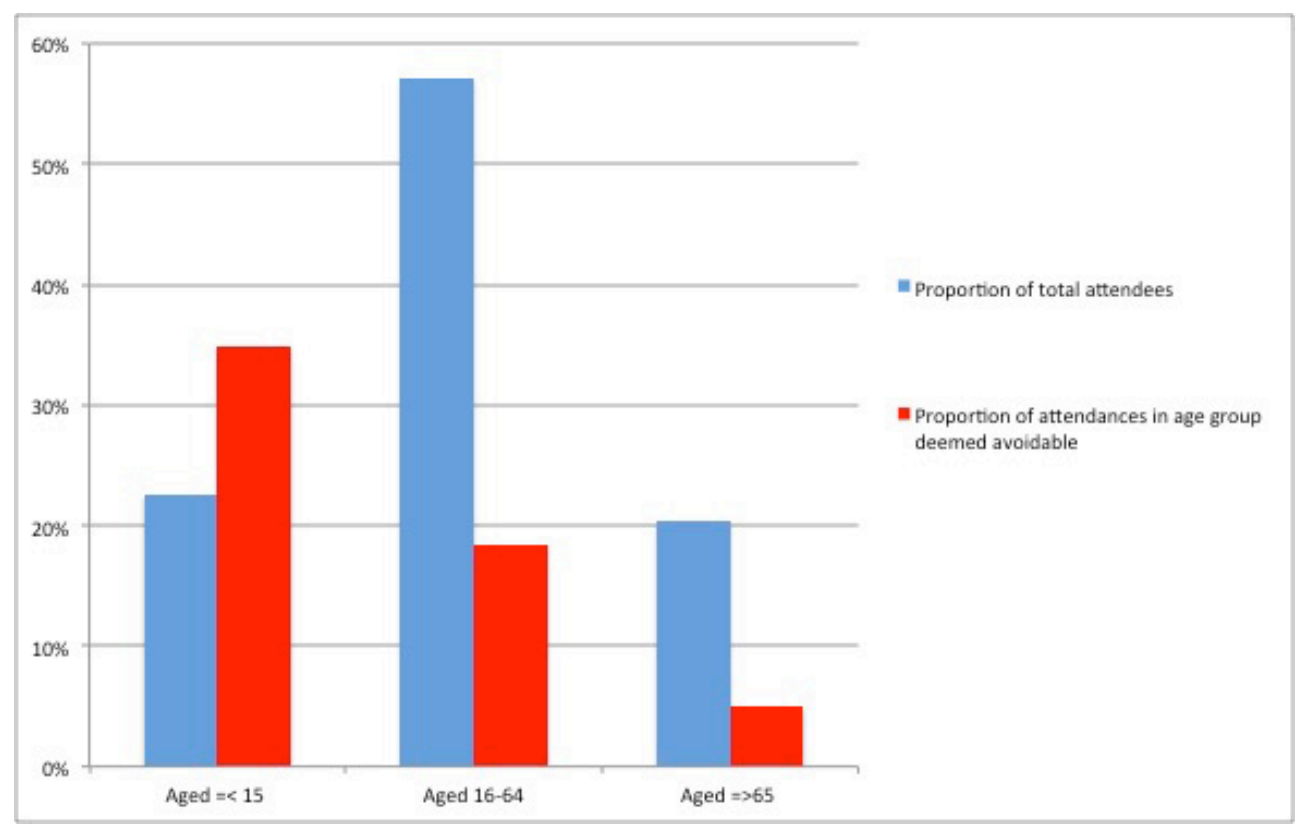

Figure 2 Bar chart showing the proportion of total attendances by age group and the proportion of avoidable attendances in each age group. 
(under 10 years) and young adults. However, another study has shown a larger proportion $(38.6 \%)$ of patients aged over 65 years to be non-urgent. ${ }^{24}$ The authors claim though that this figure is only partially comparable with that of other studies, due to variable applications of triage coding systems and different definitions and methodologies for calculating avoidability.

On the subject of age, it is important to note the differences in care between children and adults. The acute management of children is generally more conservative, with fewer invasive tests and less exposure to potential harm (eg, from ionising radiation). In the context of this study, more conservative management could give the impression of a greater proportion of AAs, although the attendances may in fact be appropriate. Conversely, it is possible that practising so-called 'defensive' medicine in the adult setting (ie, conducting investigations that may not be strictly necessary) could have given a falsely low number of AAs. In addition, it is possible that there is a greater perceived urgency in paediatric cases from parents/carers, which would lead to greater numbers of ED attendances in which no interventions are required (deemed in this study to be avoidable). We did not record perceived urgency of cases; however, this may be a factor a repeated survey in the future could assess.

The 2014 Nuffield Trust report 'Focus On: A\&E Attendances', states that the proportion of visits to the ED by patients over 65 in 2012/2013 was $21.2 \%$, comparable with the proportion reported in this study. ${ }^{25}$ It is known that people aged 65 years and over have a large impact on the urgent care system. ${ }^{25}$ The SSS reported that only $6.3 \%$ of attendances by patients aged over 65 years were avoidable, meaning that the ED is likely to be the most appropriate source of care for the vast majority of these older patients. If elderly patients are indeed increasing the pressure on the ED and contributing to the overall issue of crowding, deflection policies, as suggested in the Urgent and Emergency Care Review and other literature, ${ }^{11} 1326$ may not necessarily be the safest option.

\section{Implications for practice and future research}

The results of this study show that it is likely that a lower proportion of patients are attending EDs avoidably than previously thought. ${ }^{14}$ If this is indeed the case, then it is likely that AAs are potentially a less significant factor in the overall problem of crowding and exit block in the ED, meaning that the difficult task of redirecting avoidable patients may have limited impact. ${ }^{11} 1327$ Shifting patient care from the ED to the community could be beneficial for some patients and may slightly reduce pressure on the ED; however, this study suggests that the majority of patients (especially patients over the age of 65 years) do require the services of the ED. Any interventions attempting to redirect patients felt to be attending avoidably would be best targeted at younger patients or the parents of young children. We note that redirecting a proportion of patients would not mean an equal reduction in ED workload or expenditure. It is likely that the $19.4 \%$ of patients with AAs create a much lower proportion of workload for the ED.

Changing the health-seeking behaviours of patients in order to direct them away from the ED is challenging, and interventions have previously been shown to be ineffective. ${ }^{28} 29$ Studies claim that this is partly due to patient perceptions of increased convenience in accessing care as and when needed, the 'One Stop Shop' approach of the ED, increased quality of care and a greater ability to deal with urgent problems when compared with some other services that also deliver urgent and emergency care. ${ }^{133031}$ The RCEM STEP campaign (staffing, tariffs \& terms, exit block, primary services), devised in 2014, was developed in order to rectify significant challenges faced by EDs in four main areas, one component of which is the colocation of primary care and other relevant services with major EDs (an approach also supported by other medical royal colleges). ${ }^{32}$ Colocation of these services may be a more successful method for supporting the ED while responding to patient demand and modern healthcare-seeking behaviours. It is recommended that further research regarding colocated services and the role of GPs within the ED is carried out. Similarly, older people have been shown to require acute medical care, and therefore, there is an urgent need to develop and evaluate methods of caring for older people with acute illness that could bypass the ED, reducing pressures on departments and hospital admissions.

The SSS was designed as a pilot study and as a small-scale preliminary study and was successful. We were able to show that our study was adequately powered with regards to sample size. Involving all type 1 EDs, ideally from around the UK (rather than just England), would give more representative results on proportions of AAs. Conducting the study over a longer time period would also mitigate daily variation in factors such as staffing and attendances. Additionally, this would provide an insight into how the number of attendees and proportion of AAs fluctuates over time. Further possible correlations between AAs and other factors could also be explored in future research, such as presenting complaints, time of attendance or the use of a telephone triage system. However, a longer or more complex survey has the potential to be increasingly labour intensive for the ED staff who complete the survey.

\section{Limitations}

As with other literature in this area, the results of this study were heavily dependent on the defining criteria used for avoidability. This highlights the need for a standardised definition/defining criteria for AAs in order to increase comparability of research in this area. Until a standardised definition of avoidability is recognised, results and conclusions regarding the impact of AAs must be interpreted and acted on with caution. The criteria selected in our study were believed to be sufficiently inclusive as to ensure the formula had maximum sensitivity for excluding those who did not fulfil the criteria without compromising specificity for those who would require the care, expertise and resources of the ED.

The retrospective nature of data entry led to the possibility of bias in responding to some questions. As a result Q11 and Q12 (shown in box 1) were not used in our analysis, due to the potential for recall bias and the subjective nature of these questions. It is worth noting that some departments chose not to answer Q12 due to concerns about its validity, meaning that alongside our concerns regarding accuracy, a full data set was not available. Had this question been answered prospectively by the triage nurse or a clinician at the point of attendance, it could have been far more reliable and would have given more insight into whether attendees could be redirected at the point of triage.

Our conclusions regarding age were based on dividing patients into three broad categories representing children, adults and older patients. Our results have shown that age is likely to be a significant factor in studying AAs, and therefore we intend to divide ages into tighter bands in a follow-up, larger scale study to explore this association in more depth.

The sites used for this study were all located in England and selected on a voluntary, rather than random, basis. In the interests of time, not all type 1 departments in England could be 
invited to participate. While an increased risk of bias exists with a non-random selection, we aimed to minimise any chance of bias in responses from departments by removing subjective questions from our defining criteria. Despite the non-random selection, we believe the departments included have a sufficient range in terms of size, location and case mix to validate the results of this study. Our study aimed to capture a 'standard day' and was chosen to be midweek, at a time of year when extremes of weather would not be expected; however, daily variation in attendances is inevitable, and we recommend future studies are conducted over a longer time period to mitigate this variation.

\section{CONCLUSION}

Using our methodology for defining avoidability, we found that $19.4 \%$ of patients could be considered to be attending the ED avoidably. In the light of this figure, avoidable patients may not be contributing as significantly as was previously thought to the issue of crowding. Interventions aimed at reducing AAs would be best targeted at young patients and the parents of young children. A standardised definition of avoidability is required to further research and understanding in this area.

Acknowledgements The authors would like to thank all the departments that took part in the Sentinel Site Survey and the clinicians who completed the survey. The authors would also like to acknowledge the contribution of the following EDs in data collection: Leeds General Infirmary, St James' University Hospital Leeds, Northern General Hospital, Sheffield, St. Thomas' Hospital, London, Birmingham Children's Hospital, Royal United Hospital, Bath, Russell's Hall Hospital, Dudley, Royal Bolton Hospital, James Cook Hospital, Middlesborough, Queen Alexandra Hospital, Portsmouth, and Musgrave Park Hospital, Taunton.

Contributors Study planned by TM and SMM using data collected by the RCEM. Main data analysis conducted and submitted by TM. Consultation regarding data analysis and methodology of study with CK and CM throughout project. TM and SMM were responsible for overall content.

Funding $\mathrm{CO}$ was funded by the NIHR Collaboration for Leadership in Applied Health Research and Care Yorkshire and Humber (NIHR CLAHRC YH) (www.clahrcyh.nihr.ac.uk).

Disclaimer The views and opinions expressed are those of the author(s), and not necessarily those of the NHS, the NIHR or the Department of Health.

Competing interests None declared.

Provenance and peer review Not commissioned; externally peer reviewed.

(c) Article author(s) (or their employer(s) unless otherwise stated in the text of the article) 2018. All rights reserved. No commercial use is permitted unless otherwise expressly granted.

\section{REFERENCES}

1 Hoot NR, Aronsky D. Systematic review of emergency department crowding: causes, effects, and solutions. Ann Emerg Med 2008;52:126-36.

2 Richardson DB. Increase in patient mortality at 10 days associated with emergency department overcrowding. Med J Aust 2006;184:213-6.

3 Carter EJ, Pouch SM, Larson EL. The relationship between emergency department crowding and patient outcomes: a systematic review. J Nurs Scholarsh 2014:46:106-15.

4 Depinet HE, lyer SB, Hornung $\mathrm{R}$, et al. The effect of emergency department crowding on reassessment of children with critically abnormal vital signs. Acad Emerg Med 2014;21:1116-20.

5 QualityWatch. A\&E waiting times 2016. http://www.qualitywatch.org.uk/indicator/aewaiting-times (accessed 11 Jul 2016).

6 McHale P, Wood S, Hughes K, et al. Who uses emergency departments inappropriately and when - a national cross-sectional study using a monitoring data system. BMC Med 2013;11:258
7 O'Cathain A, Knowles E, Maheswaran R, et al. A system-wide approach to explaining variation in potentially avoidable emergency admissions: national ecological study. BMJ Qual Saf 2014;23:47-55.

8 Schull MJ, Kiss A, Szalai JP. The effect of low-complexity patients on emergency department waiting times. Ann Emerg Med 2007;49:257-64.

9 Carret ML, Fassa AC, Domingues MR. Inappropriate use of emergency services: a systematic review of prevalence and associated factors. Cad Saude Publica 2009:25:7-28.

10 Thompson MI, Lasserson D, McCann L, et al. Suitability of emergency department attenders to be assessed in primary care: survey of general practitioner agreement in a random sample of triage records analysed in a service evaluation project. BMJ Open 2013;3:e003612.

11 Nagree Y, Camarda VJ, Fatovich DM, et al. Quantifying the proportion of general practice and low-acuity patients in the emergency department. Med J Aust 2013;198:612-5

12 Honigman LS, Wiler JL, Rooks S, et al. National Study of Non-urgent Emergency Department Visits and Associated Resource Utilization. West J Emerg Med 2013;14:609-16.

13 Dinh MM, Bein KJ, Latt M, et al. Age before acuity: the drivers of demand for emergency department services in the Greater Sydney Area. Emerg Med J 2015;32:708-11.

14 NHS-England. High quality care for all, now and for future generations: transforming urgent and emergency care services in England - urgent and emergency care review end of phase 1 report, 2013. www.nhs.uk/NHSEngland/keogh-review/Documents/ UECR.Ph1Report.FV.pdf (accessed 10 Feb 2016).

15 RCEM. A\&E: more useful than people think: RCEM, 2014.

16 The king's fund. what's going on in A\&E? the key questions answered, 2017. https:// www.kingsfund.org.uk/projects/urgent-emergency-care/urgent-and-emergency-caremythbusters (accessed 2 Jul 2017).

17 Lowy A, Kohler B, Nicholl J. Attendance at accident and emergency departments: unnecessary or inappropriate? J Public Health Med 1994;16:134-40.

18 Campbell M, Machin D, Walters S. Medical statistics: a textbook for health sciences. 4 edn. Chippenham: John Wiley \& Sons, 2007.

19 NHS-England. Safer, Faster, Better: good practice in delivery urgent and emergency care. http://www.nhs.uk/NHSEngland/keogh-review/Documents/safer-faster-betterv28.pdf (accessed 20 Feb 2016).

20 Uscher-Pines L, Pines J, Kellermann A, et al. Deciding to Visit the Emergency Department for Non-Urgent Conditions: A Systematic Review of the Literature. Am J Manag Care 2013;19:47-59.

21 Cowling TE, Cecil EV, Soljak MA, et al. Access to primary care and visits to emergency departments in England: a cross-sectional, population-based study. PLoS One 2013:8:e66699.

22 David M, Schwartau I, Anand Pant H, et al. Emergency outpatient services in the city of Berlin: Factors for appropriate use and predictors for hospital admission. Eur 1 Emerg Med 2006;13:352-7.

23 Afilalo J, Marinovich A, Afilalo M, et al. Nonurgent emergency department patient characteristics and barriers to primary care. Acad Emerg Med 2004;11:1302-10.

24 Fusco M, Buja A, Furlan $\mathrm{P}$, et al. Older adults in Emergency Department: management by clinical severity at triage. Ann Ig 2014;26:409-17.

25 Blunt I. Focus on: $A+E$ attendances. The Nuffield Trust, 2014. http://www. qualitywatch.org.uk/focus-on/ae-attendances (accessed 16 Jul 2016).

26 Dent A, Hunter G, Webster AP. The impact of frequent attenders on a UK emergency department. Eur J Emerg Med 2010;17:332-6.

27 Nagree Y, Ercleve TN, Sprivulis PC. After-hours general practice clinics are unlikely to reduce low acuity patient attendances to metropolitan Perth emergency departments. Aust Health Rev 2004;28:285-91.

28 Bosmans JE, Boeke AJ, van Randwijck-Jacobze ME, et al. Addition of a general practitioner to the accident and emergency department: a cost-effective innovation in emergency care. Emerg Med J 2012;29:192-6.

29 Boeke AJ, van Randwijck-Jacobze ME, de Lange-Klerk EM, et al. Effectiveness of GPs in accident and emergency departments. Br J Gen Pract 2010;60:e378-84.

30 Göransson KE, De Waern M, Lindmarker P. Patients' pathway to emergency care: is the emergency department their first choice of care? Eur J Emerg Med 2013:20:45-50.

31 Siminski P, Cragg S, Middleton R, et al. Primary care patients' views on why they present to Emergency Departments: Inappropriate attendances or inappropriate policy? Australian Journal of Primary Health 2005;11:87-95.

32 RCEM. STEP campaign. Campaign, 2014. http://www.collemergencymed.ac.uk/ShopFloor/Policy/STEP (accessed 18 Feb 2015). 